\title{
Nocardia cyriacigeorgica bacteraemia presenting with cytomegalovirus disease and rapidly fatal pneumonia in a renal transplant patient: a case report
}

\author{
Simon Namnyak ${ }^{1 *}$, Mashuk Uddin ${ }^{2}$ and Nadia Ahmod $^{3}$
}

\begin{abstract}
Introduction: Nocardia cyriacigeorgica bacteraemia has been described in the setting of profound immunodeficiency in only two previous case reports. In both instances, diagnosis was rapidly facilitated by 165 rRNA gene sequencing of blood culture isolates. To the best of our knowledge, we believe that our case is the first presentation of $N$. cyriacigeorgica bacteraemia associated with acute cytomegalovirus disease in a kidney transplant recipient, which was then followed by severe and fatal pneumonia only seven days later.

Case presentation: We present the case of a 73 -year-old Caucasian woman, a renal transplant recipient, with peripheral vascular disease, hypertension, osteoporosis and vascular dementia who was diagnosed with septicemia and pneumonia. In spite of appropriate anti-microbial therapy for nocardial sepsis, she developed severe pneumonia and acute renal failure.

Conclusion: This case illustrates a potential for disseminated nocardial infection to produce clinical syndromes that may be indistinguishable from acute cytomegalovirus disease. An atypical presentation (pneumonia and renal failure) of a rare disease (nocardial septicemia) in the setting of renal transplantation is discussed. This case illustrates that the possibility of severe cytomegalovirus disease should be considered in renal transplanted patients diagnosed with nocardial septicemia who subsequently develop severe sepsis, pneumonia, and renal failure. Molecular diagnosis should readily be available to assist with the prompt diagnosis and treatment of these infections in renal transplant patients.
\end{abstract}

\section{Introduction}

Nocardia cyriacigeorgica bacteraemia has been described in the setting of profound immunodeficiency in only two previous case reports [1,2]. Our case is unusual due to its co-existence with cytomegalovirus (CMV) disease, renal transplantation, and acute rapidity and severity of the subsequent fatal pneumonia. Although there is a link between nocardial septicemia and various medical conditions, its association with acute CMV disease in this setting remains elusive [3,4]. Disseminated nocardiosis and acute CMV disease are known to induce an assemblage of clinical syndromes which are non-specific,

\footnotetext{
* Correspondence: Simon.Namnyak@bhrhospitals.nhs.uk

'Department of Medical Microbiology, Queen's Hospital, Barking, Havering and Redbridge University Hospitals NHS Trust, Romford, RM7 OAG, Essex, UK Full list of author information is available at the end of the article
}

requiring rapid, sensitive and specific microbiological techniques to delineate one from the other. In summary, we describe a novel and highly unusual case in which the diagnosis of nocardial septicemia co-existed with acute CMV disease only diagnosed after death.

\section{Case Presentation}

Our patient was a 73-year-old Caucasian woman with a transplanted kidney, peripheral vascular disease, hypertension, osteoporosis and vascular dementia who presented to our hospital with a 10-day history of nonproductive cough, vomiting, anorexia, and fever and was non-specifically unwell. The family doctor had recently treated her with trimethoprim for a urinary tract infection. She had received a kidney transplant from her sister 11 years previously due to polycystic kidney disease

\section{() Biomed Central}


and she was receiving azathioprine (50 $\mathrm{mg}$ once daily), cyclosporine (75 mg twice daily) and prednisolone (7.5 mg once daily).

On examination, our patient was confused, restless and pyrexial with a temperature of $38.4 \llbracket C$, respiration rate of 24 breaths/min, blood pressure $111 / 72 \mathrm{mmHg}$, and reduced air entry to both lung bases. An echocardiogram showed sinus tachycardia of 129 beats $/ \mathrm{min}$. She appeared cachexic and dehydrated. Hematological and biochemical investigations revealed hemoglobin $9.7 \mathrm{~g} / \mathrm{dl}$, white cell count $16.0 \times 10^{9} / \mathrm{L}$ (neutrophils $14.4 \times 10^{9}$ /L), sodium $136 \mathrm{mmol} / \mathrm{L}$, potassium $5.8 \mathrm{mmol} / \mathrm{L}$, urea $49.7 \mathrm{mmol} / \mathrm{L}$, creatinine $447 \mu \mathrm{mol} / \mathrm{L}$, bilirubin $18 \mu \mathrm{mol} /$ $\mathrm{L}$, alanine aminotransferase $60 \mathrm{i} \mathrm{u} / \mathrm{L}$, alkaline phosphatase $92 \mathrm{i} \mathrm{u} / \mathrm{L}$, total protein $60 \mathrm{~g} / \mathrm{L}$, albumin $25 \mathrm{~g} / \mathrm{L}$, and C-reactive protein (CRP) $315 \mathrm{mg} / \mathrm{L}$. A clotted blood sample and ethylene-diamine-tetra-acetic acid (EDTA) whole blood sample for CMV quantitative polymerase chain reaction (PCR) were obtained on the day of death. The clotted sample was positive for CMV immunoglobulin M (IgM) antibodies but negative for CMV immunoglobulin G (IgG) antibodies, and the EDTA blood sample for CMV PCR yielded 11, 899 copies/mL consistent with active CMV disease at the limit of sensitivity of the test at 500 copies $/ \mathrm{mL}$.

A chest X-ray revealed a right lower lobe infiltrate and severe kyphoscoliosis. An arterial blood gases analysis on air showed $\mathrm{pH} 7.424$, partial carbon dioxide $\left(\mathrm{pCO}_{2}\right)$ $3.31 \mathrm{kPa}$, partial oxygen $\left(\mathrm{pO}_{2}\right) 32.16 \mathrm{kPa}$, saturation of peripheral oxygen $\left(\mathrm{SpO}_{2}\right) 99.6 \%$ and base excess (BE) $-6.5 \mathrm{mmol} / \mathrm{L}$. A clinical diagnosis of communityacquired pneumonia was made and given a CURB-65 score of $3 / 5$, giving a prediction of $17 \%$ risk of death. Our patient was known to be mildly allergic to penicillin and was therefore started on empirical intravenous antibiotics with imipenem $500 \mathrm{mg}$ every 12 hours to treat possible multiply-resistant Gram-negative bacilli associated with urosepsis, and $1000 \mathrm{~mL}$ of sodium chloride intravenous infusion $0.9 \%$ was given over eight hours and repeated as appropriate to correct dehydration. Although she remained apyrexial with normal hemodynamic variables for a few more days, her inflammatory markers remained markedly raised and renal function continued to deteriorate gradually despite antibiotics, intravenous fluids and renal support.

On admission to hospital, one set of BacT/ALERT 3D (bioMérieux, Inc., Durham, North Carolina, USA) aerobic and anaerobic blood cultures were collected. After four days of incubation, the aerobic bottle demonstrated long thin Gram positive rod shaped bacterium, identified as Nocardia species, sensitive to doxycycline and imipenem, resistant to penicillin, erythromycin, trimethoprim, levofloxacin, clindamycin, rifampicin, teicoplanin and vancomycin based on British Society for
Antimicrobial Chemotherapy (BSAC) disk diffusion susceptibility testing. A diagnosis of disseminated nocardiosis was subsequently made and imipenem was continued.

Our patient's clinical condition deteriorated rapidly on day six, developing severe acidotic breathing, reduced level of consciousness (Glasgow Coma Scale 3/15) and acute oliguria, despite adequate fluid resuscitation. Arterial blood gases analysis on $2 \mathrm{~L}$ of oxygen showed $\mathrm{pH}$ 7.121, $\mathrm{pCO}_{2} 8.41 \mathrm{kPa}, \mathrm{pO}_{2} 8.77 \mathrm{kPa}, \mathrm{SpO}_{2} 85.3 .6 \%$ and $\mathrm{BE}-10.1 \mathrm{mmol} / \mathrm{L}$. Hematological and biochemical investigations revealed hemoglobin $6.8 \mathrm{~g} / \mathrm{dL}$, white cell count $10.7 \times 10^{9} / \mathrm{L}$ (neutrophils $9.8 .4 \times 10^{9} / \mathrm{L}$ ), sodium $136 \mathrm{mmol} / \mathrm{L}$, potassium $5.4 \mathrm{mmol} / \mathrm{L}$, urea $43.5 \mathrm{mmol} / \mathrm{L}$, creatinine $312 \mu \mathrm{mol} / \mathrm{L}$, bilirubin $6 \mu \mathrm{mol} / \mathrm{L}$, alkaline phosphatase $83 \mathrm{i} \mathrm{u} / \mathrm{L}$, total protein $40 \mathrm{~g} / \mathrm{L}$, albumin $14 \mathrm{~g} /$ $\mathrm{L}$, and CRP $178 \mathrm{mg} / \mathrm{L}$. A chest physician consultation confirmed that our patient was in severe respiratory failure and had severe pneumonia, but was unlikely to benefit from the use of high dose co-trimoxazole and/or valganciclovir respectively to cover for possible Pneumocystis jirovecii or CMV infection, and critical care support and assisted ventilation. The patient died on day seven after admission, and her family requested that an autopsy not be performed.

The blood culture isolate was confirmed as $N$. cyriacigeorgica at the Molecular Identification Services Unit, Centre for Infections, Health Protection Agency, London, by using partial sequencing of the $16 \mathrm{~S}$ rDNA and cluster analysis of the gyraseB gene (Figure 1).

\section{Discussion}

The incidence of nocardial infections is believed to be on the rise worldwide as a result of a growing immunecompromised population and improved methods for pathogen isolation and molecular identification [5].

Disseminated nocardiosis is frequently characterized by pulmonary involvement in over $68 \%$ of patients, while isolation of the organism, and particularly $N$. cyriageorgica, from blood is very rare [6] even in the presence of severe immune-suppression, cancer and transplantation [2]. These observations have generally remained unexplained in the literature, although it has been suggested that if nocardia infection is clinically suspected, then efforts to optimize the recovery of the micro-organisms in a Bactec blood culture system should be used, with prolonged incubation beyond the usual five days and with frequent and terminal subculturing [5]. In the present case, blood cultures incubated in a Bactec blood culture system yielded growth in four days, but unfortunately a sputum sample was not obtained to confirm presence of Nocardia in her lung as right lower lobe infiltrate was identified with radiology. 


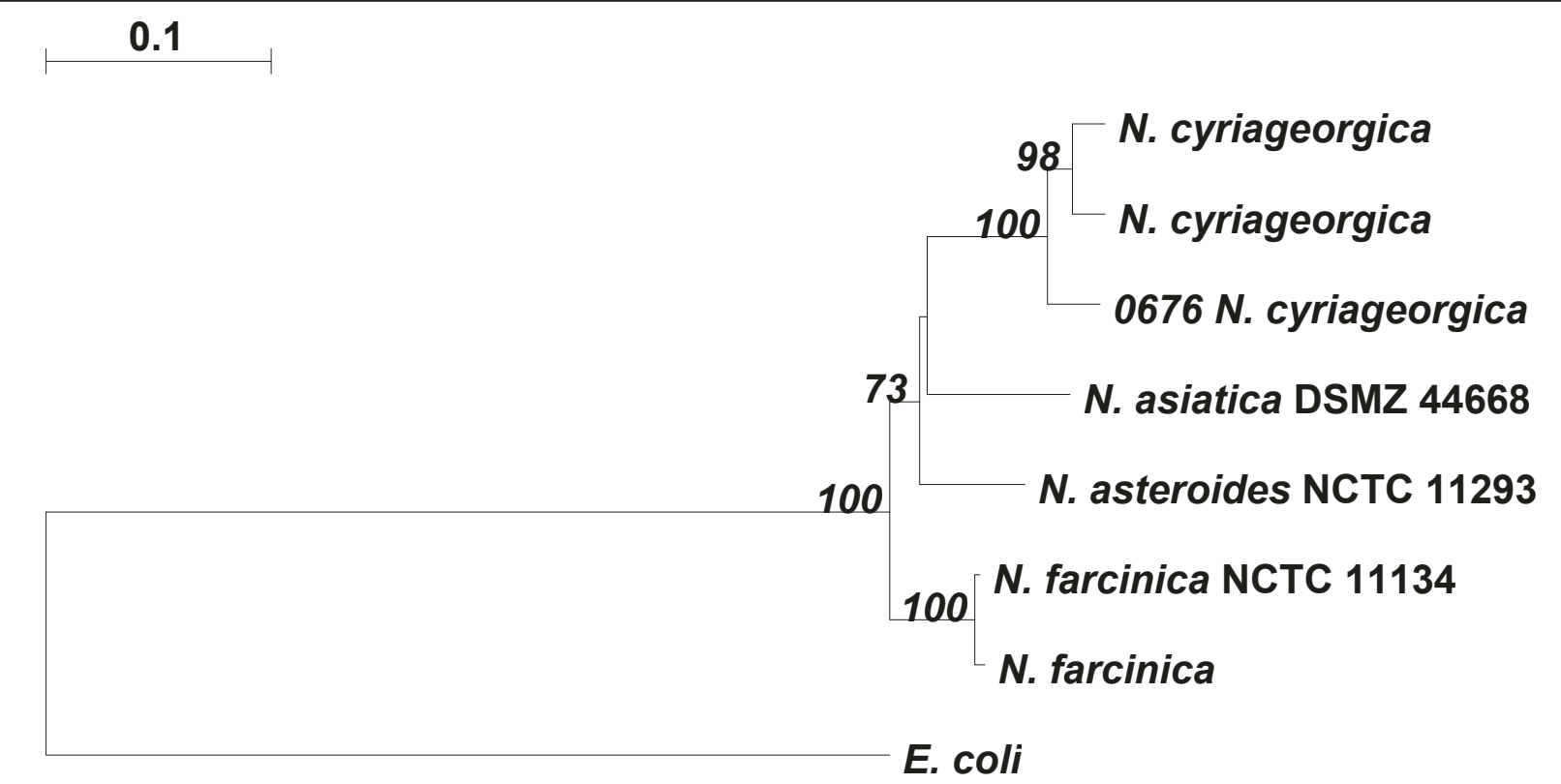

Figure 1 The tree shows the clustering of Nocardia species based on the similarity of their gyr gene sequences. The scale bar is a measure of the evolutionary distance between species based on the length of the branches. Case Report isolate of $\mathrm{N}$. cyriageorgica is number 0676.

Numerous new species of the $N$. asteroides complex have been recently described, including $N$. cyriacigeorgica $[7,8]$. This species has been rarely reported and overlooked in human infections so far, since molecular techniques for species identification have not been available [9]. Thus, the present isolate was initially identified only as Nocardia species by the API system.

Our patient was treated with intravenous imipenem to which the isolate was sensitive but there was no clinical improvement noted. Until now, no study has established a correlation between the results obtained in vitro and the clinical outcome of the patients under treatment with imipenem [10]. Previous studies have suggested clinical response of patients treated with cotrimoxazole was higher than those treated with other antibiotics [6].

In a recent prospective study of 471 consecutive renal transplant recipients, both asymptomatic CMV infection and CMV disease were identified as independent risk factors for overall mortality beyond 100 days post-transplantation [11]. Our patient presented with fever, anorexia, vomiting, coughing, lymphopenia and elevated liver enzymes, symptoms consistent with CMV disease as confirmed by significantly elevated CMV viral load at diagnosis [12]. It is debatable whether our patient could have benefited from early prophylaxis and treatment with ganciclovir if CMV PCR results were known in the early stages of her disease [12].

Although the association of bacteraemic nocardiosis with systemic steroids are described, to the best of our knowledge this is the first case in which nocardiosis is linked to acute CMV disease. There are conflicting reports on whether or not the intensity of immune-suppression in kidney transplant recipients is associated with the risk of CMV infections [11] and therefore further studies are needed to identify any risk factors for CMV infections in kidney transplant recipients to enable prevention strategies for CMV infection to be reconsidered.

\section{Conclusion}

$N$. cyriacigeorgica septicemia and pneumonia in a kidney transplant recipient, co-existing with CMV disease, is rarely reported and may be difficult to diagnose with traditional microbiological methods. New molecular typing schemes are now available to identify new species and assist with the management of patients with invasive disease. Co-existing CMV disease and infections should be diagnosed promptly with CMV PCR to assist with the early treatment and/or prophylaxis with valganciclovir. This case contributes to the spectrum of diseases that may be seen in immune-suppressed patients after kidney transplantation.

\section{Consent}

Written informed consent was obtained from the patient's next-of-kin for publication of this case report and any accompanying images. A copy of the written consent is available for review by the Editor-in-Chief of this journal. 


\section{Author details}

'Department of Medical Microbiology, Queen's Hospital, Barking, Havering and Redbridge University Hospitals NHS Trust, Romford, RM7 OAG, Essex, UK. ${ }^{2}$ Department of Medicine, Queen's Hospital, Barking, Havering and Redbridge University Hospitals NHS Trust, Romford, RM7 OAG, Essex, UK. ${ }^{3}$ Molecular Identification Services, Centre for Infections, Health Protection Agency, Colindale, London NW9 5HT, UK.

\section{Authors' contributions}

SN, lead and corresponding author, managed this patient clinically, and helped to draft the manuscript and performed the literature review. MU managed the patient clinically, and helped to draft the manuscript and performed the literature review. NA identified the isolate as N. cyriacigeorgica by using partial sequencing of the $16 \mathrm{~S}$ rDNA and cluster analysis of the gyraseB gene, and helped to draft the manuscript and perform the literature review. All authors read and approved the final manuscript.

\section{Competing interests}

The authors declare that they have no competing interests.

Received: 2 September 2010 Accepted: 23 June 2011

Published: 23 June 2011

\section{References}

1. Schlaberg R, Huard RC, Della-Latta P: Nocardia cyriacigeorgica, an emerging pathogen in the United States. J Clin Microbiol 2008, 46:265-273.

2. Elsayed S, Kealey A, Coffin CS, Read R, Megran D, Zhang K: Nocardia cyriacigeorgica septicaemia. J Clin Microbiol 2006, 44:280-282.

3. Yassin AF, Steiner U: Nocardia cyriacigeorgici sp. nov. Int J Syst Evol Microbiol 2001, 51:1419-1423.

4. Conville PS, Witebsky FG: Analysis of multiple differing copies of the $16 \mathrm{~S}$ copies of the 16S rRNA gene in five clinical isolates and three type strains of Nocardia species, and implications for species assignment. J Clin Microbiol 2007, 45:1146-1151.

5. Brown-Elliott BA, Brown JM, Conville PS, Wallace RJ Jr: Clinical and laboratory features of the Nocardia spp. based on current molecular taxonomy. Clin Microbiol Rev 2006, 19:259-282.

6. Kontoyiannis DP, Ruoff K, Hooper DC: Nocardia bacteremia. Report of 4 cases and review of the literature. Medicine 1998, 77:255-267.

7. Roth AS, Andrees R, Kroppenstedt M, Harmsen D, Mauch H: Phylogeny of the genus Nocardia based on reassessed 16S rRNA gene sequences reveals underspeciation and division of strains classified as Nocardia asteroides into three established species and two unnamed taxons. J Clin Microbiol 2003, 41:851-856.

8. Wauters GV, Avesani J, Charlier M, Janssens M, Vaneechoutte M, Delmee M: Distribution of Nocardia species in clinical samples and their routine rapid identification in the laboratory. J Clin Microbiol 2005, 43:2624-2628.

9. Akcaglar S, Yilmaz E, Heper Y, Alver O, Akalin H, Ener B, Tore O, Ersoy C, Imamoglu S: Nocardia cyriacigeorgica: pulmonary infection in a patient with Basedow-Graves disease and a short review of reported cases. Int J Infect Dis 2008, 12:335-338.

10. Barnaud G, Deschamps C, Manceron V, Mortier E, Laurent F, Bert F, Boiron P, Vinceneux P, Branger C: Brain abscess caused by Nocardia cyriacigeorgica in a patient with human immunodeficiency virus infection. J Clin Microbiol 2005, 43:4895-4897.

11. Helanterä I, Lautenschlager I, Koskinen P: Prospective follow-up of primary CMV infections after 6 months of valganciclovir prophylaxis in renal transplant recipients. Nephrol Dail Transplant 2009, 24:316-320.

12. Arthurs SK, Eid AJ, Pedersen RA, Kremers WK, Cosio FG, Patel R, Razonable R: Delayed-onset primary cytomegalovirus disease and the risk of allograft failure and mortality after kidney transplantation. Clin Infect Dis 2008, 46:840-846.

doi:10.1186/1752-1947-5-228

Cite this article as: Namnyak et al: Nocardia cyriacigeorgica bacteraemia presenting with cytomegalovirus disease and rapidly fatal pneumonia in a renal transplant patient: a case report. Journal of Medical Case Reports 2011 5:228.

\section{Submit your next manuscript to BioMed Central and take full advantage of:}

- Convenient online submission

- Thorough peer review

- No space constraints or color figure charges

- Immediate publication on acceptance

- Inclusion in PubMed, CAS, Scopus and Google Scholar

- Research which is freely available for redistribution 